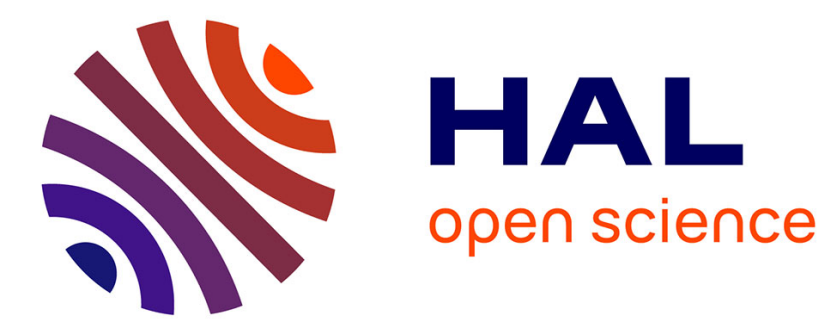

\title{
Du bricolage en général et des dictionnaires de langue en particulier
}

Jean-Philippe Dupuy

\section{To cite this version:}

Jean-Philippe Dupuy. Du bricolage en général et des dictionnaires de langue en particulier. 2009. hal-00448763

\section{HAL Id: hal-00448763 \\ https://hal.science/hal-00448763}

Preprint submitted on 20 Jan 2010

HAL is a multi-disciplinary open access archive for the deposit and dissemination of scientific research documents, whether they are published or not. The documents may come from teaching and research institutions in France or abroad, or from public or private research centers.
L'archive ouverte pluridisciplinaire HAL, est destinée au dépôt et à la diffusion de documents scientifiques de niveau recherche, publiés ou non, émanant des établissements d'enseignement et de recherche français ou étrangers, des laboratoires publics ou privés. 
Jean-Philippe Dupuy

Université de Bourgogne

\section{Du bricolage en général et des dictionnaires de langue en particulier.}

Mots-clés : dictionnaire, langue, linguistique, idéologie, lexicographie.

Résumé : Si le dictionnaire de langue apparaît au grand public comme une norme intangible, il procède en fait d'une série d'ajustements, de décisions subjectives, de choix empiriques voire idéologiques: ce sont précisément ces hésitations qui nous intéressent, et qui font de la dictionnairique un art plus qu'une science. Qu'il s'agisse de déterminer la nomenclature, d'en organiser la structure, de synthétiser le sens d'un vocable ou d'en sélectionner les emplois attestés, le lexicographe procède nécessairement à des choix puisque la langue qu'il cherche à reconstruire est un pur artefact, un produit de synthèse censé rendre compte d'un hypothétique «français standard » : mais il n'est pas de choix neutre ou innocent, et c'est la société, avec ses tabous et ses partis pris, que l'on pourra lire entre les lignes du dictionnaire.

\section{On fiddling about with words in general and on language dictionaries in particular.}

Keywords: dictionary, language, linguistics, ideology, lexicography.

Abstract: If language dictionaries appear as an invariable norm to the general public, they are in actual fact the result of a series of adjustments, of subjective decisions and of empirical or even ideological choices. Those very hesitations are of prime interest and turn dictionary science into an art rather than a science proper. Whether the task of lexicographers consists in determining the word list, in organizing its structure, in synthesizing the meaning of terms, or in selecting their registered uses, they necessarily need to make personal choices since the language they are trying to reconstruct is nothing but an artefact, the synthesis of a so-called "standard Language". But there is no such thing as a neutral or unbiased choice, so that eventually it is the portrait of a society, along with its taboos and prejudices, which will appear between the lines of a dictionary. 
Jean-Philippe Dupuy ${ }^{*}$

Université de Bourgogne

\section{Du bricolage en général et des dictionnaires de langue en particulier}

Le thème du XXXI ${ }^{\mathrm{e}}$ congrès de l'APLIUT, «le dictionnaire dans tous ses états », ne manque pas d'ambition! Un simple coup d'œil sur la production éditoriale mensuelle nous permet de constater en effet la variété des sujets embrassés par les dictionnaires ; il n'est de domaine, d'activité, de loisir, de spécialité qui n'ait son dictionnaire: boulangerie, photo numérique, environnement, couleurs, langue, médecine, peinture, animaux, plantes sauvages, vins... tout est matière dictionnairique ! À y regarder de plus près, les buts de ces ouvrages diffèrent sensiblement: définir, bien sûr (dictionnaires juridiques, médicaux, financiers, etc.), traduire (dictionnaires bilingues, des sigles...), mais aussi simplement répertorier (les chats, les plantes, des noms de famille, les citations, etc.) ou simplement évoquer, tels les dictionnaires amoureux (des langues, de la Grèce, du vin, de la gastronomie, de Venise, et même un dictionnaire amoureux des dictionnaires (Rey 2010)!).

Comment expliquer le succès des dictionnaires, cette dicomania ${ }^{1}$ dont on parlait déjà il y a une dizaine d'années ? Certes, on ne peut ignorer le goût respectable du public pour le savoir, d'autant plus que l'étiquette de dictionnaire apporte des connotations de sérieux et d'exhaustivité à quelque chose qui peut très bien d'ailleurs ne présenter aucune de ces deux qualités ; mais c'est avant tout, nous semble-t-il, le succès d'une forme: tous les dictionnaires que l'on vient de mentionner ont en effet en commun un mode de classement alphabétique dont l'intérêt principal est de favoriser le discontinu : si le rédacteur du dictionnaire en tire avantage (la structure alphabétique tient lieu de problématique générale), le lecteur y trouve également son compte, le fléchage alphabétique le dispensant d'un parcours plus lent à travers la complexité d'une pensée.

Afin de réduire le propos, nous nous intéresserons simplement aux dictionnaires de langue (Larousse, Robert, par exemple), à ces dictionnaires de mots que l'on opposait autrefois aux dictionnaires de choses, encyclopédies ou dictionnaires dits universels. Comme le constate Alain Rey, il ne semble pas qu'il y ait grand-chose à

Agrégé de Grammaire et Docteur en Sciences de Langages, Jean-Philippe Dupuy a d'abord enseigné en collège et lycée, avant de rejoindre l'Université de Bourgogne (UFR Lettres, puis IUT de DIJON) où il donne des cours de littérature, linguistique, informatique et assure les fonctions de Directeur des Etudes. <jean-philippe.dupuy@u-bourgogne.fr>.

dictionnaires ».

Les Cahiers de l'APLIUT - Vol. XXIX Nº 1 - FÉVRIER 2010 - ISSN 0248-9430 
en dire! Car le dictionnaire est à la fois un objet d'évidence - sous prétexte qu'on l'a toujours connu auprès de soi, on a l'impression qu'il a toujours existé - et un objet symbolique : il représente le savoir par opposition à la croyance ${ }^{2}$ et semble nous révéler la vérité des mots et du monde ; c'est LE livre de référence qui tranche de façon incontestable: ce mot «existe-t-il»? Que veut-il dire ? Comment s'orthographie-t-il ?

Dès lors, il faut suivre Georges Perec et interroger l'habituel, l'infra-ordinaire... On découvre alors que les dictionnaires ont une histoire (ils n'ont pas toujours existé, leur structure a varié, ils sont perméables dans leur présentation et leur contenu aux goûts de l'époque); qu'ils révèlent parfois une idéologie; que ce sont aussi des marchandises, des produits qui, pour la plupart, doivent être rentables. Bref les dictionnaires n'ont pas «la belle simplicité des lettres de l'alphabet» (Barthes 1985: 228), ce sont des constructions humaines, imparfaites, non exemptes d'erreurs; ils demeurent, malgré les progrès de la lexicologie, des bricolages au sens de Lévi-Strauss ${ }^{3}$.

Contrairement à ce qu'on peut croire, il y a donc beaucoup à dire sur les dictionnaires de langue, trop même, et l'on va limiter le propos à quelques considérations introductives qui ne visent ni l'exhaustivité, ni l'originalité : esquisser l'histoire des dictionnaires, s'interroger sur leur confection et plus précisément envisager les nécessaires questions que se pose tout dictionnariste.

\section{Survol historique}

L'idée de faire un recueil un tant soi peu exhaustif du lexique d'une langue ne va pas de soi : certaines conditions linguistiques, culturelles, politiques paraissent nécessaires ; c'est en tout cas une idée récente ( $17^{\mathrm{e}}$ siècle) : auparavant, les ancêtres du dictionnaire ressemblent plutôt soit à un recueil de curiosités ${ }^{4}$, soit à un outil d'explicitation (les gloses inscrites dans les marges des manuscrits d'Aristote), soit à un outil de traduction: les glossaires de Reichenau ou de Kassel ( $8^{\mathrm{e}}$ siècle) permettent ainsi de retrouver le sens de certains mots latins difficiles ou rares, devenus incompréhensibles aussi bien pour les étudiants que pour les clercs; entre le $16^{\mathrm{e}}$ et le $18^{\mathrm{e}}$ siècle, le développement des voyages, du commerce et des études favorise de même le succès du Dictionarium d'Ambrogio Calepino : bilingue puis quadrilingue, cet ouvrage, qui permettra soixante-dix ans plus tard de jongler entre

2 Cf. Aristide Quillet qui affirme en 1934, dans sa Préface au Dictionnaire encyclopédique Quillet : «La Bible d'aujourd'hui, c'est le dictionnaire ».

3 Dans un passage célèbre de La pensée sauvage (1962), Claude Lévi-Strauss évoque la pensée mythique : au contraire de la pensée scientifique, la pensée mythique bricole, elle fait avec ce qu'elle a (matériaux, outils) ; elle prend ce qui lui tombe sous la main et elle se construit au gré des opportunités.

$4 \quad$ Varron, par exemple, dans son De lingua latina, fournit de nombreuses étymologies fantaisistes en plus de réflexions sur le fonctionnement de la langue. 
onze langues, sera réédité jusqu'à deux cents fois ! En France, l'édit de VillersCotterets (qui impose en 1539 le français comme langue du droit et de l'administration française) est immédiatement suivi de la publication des deux dictionnaires bilingues de Robert Estienne, le Dictionnaire latin-français et le Dictionnaire français-latin; il faudra attendre 1606 pour que Jean Nicot dans son Thrésor de la langue françoyse tant ancienne que moderne relègue le latin à un rôle subsidiaire : on passe d'un outil pratique permettant de traduire à un objet de savoir qui cherche à englober et définir la langue prise en compte pour elle-même. Dès lors, la voie est toute tracée pour que paraissent les trois premiers grands dictionnaires français, le Dictionnaire françois contenant les mots et les choses de Pierre Richelet (1680), le Dictionnaire universel de Furetière (1690) et, quatre ans plus tard, le Dictionnaire de l'Académie française.

Que nous apprend ce rapide survol des débuts du dictionnaire ? En premier lieu, que les premiers dictionnaires étaient des dictionnaires bilingues : on a cherché à traduire avant d'entreprendre de définir et de décrire ; la démarche pragmatique et utilitaire a précédé l'entreprise lexicographique systématique. On peut observer d'autre part que la naissance des dictionnaires de langue a été favorisée par des facteurs linguistiques et extralinguistiques :

- l'édit de Villers-Cotterets a donné à la langue française sa légitimité ;

- la littérature française des $16^{\mathrm{e}}$ et $17^{\mathrm{e}}$ siècles a permis à la langue de faire ses preuves artistiques : lister les mots, c'est en effet faire l'éloge d'une langue qui n'est plus considérée seulement comme un outil de communication mais comme une possible pratique artistique méritant d'être définie et décrite ;

- le $17^{\mathrm{e}}$ siècle est marqué par le rationalisme et la pensée cartésienne qui cherchent à classer, mathématiser, organiser ;

- à travers le Dictionnaire de l'Académie française se distingue l'affirmation d'un pouvoir politique monarchique centralisé qui veut affirmer sa puissance en donnant l'image d'un pays uni derrière sa langue.

On n'insistera pas sur l'évolution plus récente des dictionnaires: notons simplement que dans leur forme même, dans leur contenu, dans leur ambition, ils traduisent l'époque qui les voit naître : l'Encyclopédie de Diderot est à la hauteur de l'ébullition intellectuelle du $18^{\mathrm{e}}$ siècle; le positivisme d'Auguste Comte transparaît dans le Littré derrière le goût du fait et le recours aux citations ; le Larousse, qui propose une nomenclature plus riche et s'adresse à un public moins cultivé révèle la pensée éclairée de son siècle en s'offrant comme outil de vulgarisation républicaine; les dictionnaires du $20^{\mathrm{e}}$ siècle consacrent le triomphe de la mécanisation et de l'informatisation avec, pour le Trésor de la Langue française informatisé, un corpus considérable de 450000 entrées.

On le voit, le dictionnaire est d'abord le produit d'une époque, le résultat d'une culture; dans les objets décrits aussi bien que dans les méthodes de description, il révèle ses relations au monde. Quand on examine son histoire, on constate ses 
balbutiements, ses hésitations : quel public viser ? Quel registre de langue décrire ? Faut-il céder à la tentation encyclopédique ? Convient-il de faire la part belle à l'étymologie ? Autant de questions que tout dictionnariste se pose, et qu'il nous faut maintenant examiner.

\section{L'activité dictionnairique}

L'activité dictionnairique peut se résumer en quatre verbes : choisir, structurer, définir, illustrer.

\section{Choisir}

Choisir les entrées qui figureront dans le dictionnaire, c'est déterminer la nomenclature «qui normalise une liste finie d'éléments dans l'ensemble non fini du lexique » (Collinot 1997 : 54). Aucun dictionnaire ne peut prétendre en effet à l'exhaustivité puisque, à la différence d'une langue artificielle, la langue naturelle est non finie : tout locuteur dispose en effet d'une faculté de créativité lexicale qui lui permet par exemple, à partir du sigle IUT, de créer les mots iutien, post-iutien, pré-iutien, etc. Par ailleurs, pour des raisons pratiques le plus souvent, le dictionnariste ne cherche pas à inclure tous les termes attestés, mais sélectionne $n$ entrées : 18000 dans le premier dictionnaire de l'Académie, 60000 dans le Petit Robert et le Petit Larousse, 100000 dans le TLF parmi les 200000 à 500000 mots circonscrivant à peu près la langue française (auxquels il faudrait ajouter un million de termes techniques).

Pour déterminer cette nomenclature, il faut d'abord se demander si l'on cherche à décrire (rendre compte de l'usage) ou à prescrire (fixer une norme). Aujourd'hui, seul le Dictionnaire de l'Académie française joue un rôle prescripteur ; les autres dictionnaires ont, plus modestement, un rôle descriptif ${ }^{5}$ et rendent compte d'un usage : ils ne nous disent pas ce qu'on peut ou ce qu'on doit dire, mais recensent simplement ce qu'on dit. Le problème, l'ambiguïté fondamentale des dictionnaires de langue est que le public voit cette description de l'usage comme une norme et prend pour modèle ce qui n'est qu'un reflet des pratiques langagières.

Comment déterminer l'usage d'une langue ? Choisir un corpus, lui appliquer un filtre statistique, et conserver les vocables utilisés au moins $n$ fois sembleraient suffire.

Hélas, la composante statistique ne permet pas de fixer la nomenclature ! Elle conduirait en effet à ce que le dictionnaire rende compte des mots les plus fréquents - et donc les mieux connus - et ignore les mots rares, ceux pour lesquels il serait le plus utile ! Les simples données quantitatives ne suffisent donc pas : la

5 Alors que la nomenclature se veut descriptive (elle enregistre des usages), la graphie de l'entrée du dictionnaire a une valeur prescriptive: elle impose une norme orthographique, et ne présente pas les variantes possibles (sinon, on trouverait *vraissemblable, *éthymologie, *intérrêt...) ; idem pour la transcription phonétique. 
constitution de la nomenclature repose aussi sur du qualitatif, une échelle de valeurs plus ou moins intériorisée qui ferait préférer un mot utilisé très rarement chez Proust à un vocable plus fréquent mais d'un registre de langue moins soutenu ${ }^{6}$ ou rejeté pour des raisons idéologiques.

Plus encore, Marie-Hélène Drivaud, directrice éditoriale du Petit Robert, insiste sur la nécessité de prendre en compte le contexte d'emploi : «Certains [mots], pourtant très présents, n'ont pas été retenus parce qu'ils sont liés à une personne ou un événement particulier et ne sortent pas de ce cadre là ; "Bravitude", par exemple, n'a pas de fonctionnement normal. Il est toujours cité en référence à la phrase de Ségolène Royal. On ne l'a pas retenu. » Pas plus que le fameux "bling bling" évoqué à propos de Nicolas Sarkozy... ${ }^{7}$.

Quant au choix du corpus de référence, il est évidemment problématique : quelle extension diachronique lui donner ? Quelle extension géographique ? (se limitera-ton à un français standard ou intégrera-t-on les spécificités du patois bourguignon ou des dialectes antillais) ? Faut-il y intégrer des sous-corpus spécialisés (langue «jeune », sms, technique, argot, verlan...) ? Et que faire des non attendus comme *pallier à, *au jour d'aujourd'hui ? Si l'on ajoute à cela des considérations marchandes ou pratiques (taille, prix), on voit que le choix de la nomenclature relève plus de l'art du jongleur ou de l'équilibriste que de la science implacable du lexicologue.

\section{Structurer}

Là encore, les choix effectués en matière de structure n'ont rien d'évident ni d'impératif : examinons simplement trois problèmes :

Le premier problème est celui du mot, terme suspect, vague, peu scientifique: combien de mots dans pied-d'alouette? Dans chair de poule? Dans pied au plancher? À ce vieux problème de lexicologie, des auteurs multiples (Pottier, Benvéniste, Martinet, Mitterand) ont apporté des solutions variées, l'idée de base étant que l'unité graphique est insuffisante pour identifier l'unité lexicale: on parlera alors par exemple, avec Pottier (1974), de lexie simple, composée (piedd'alouette), complexe (chair de poule, pied au plancher), de groupe de mots (le cheval de Jean)... Mais une fois réglé le problème lexicologique survient la difficulté proprement dictionnairique : comment présenter les lexies complexes ? Alain Rey (1977 : 23) revient sur l'exemple de la pomme de terre: en général, les dictionnaires présentent une entrée pomme, une entrée terre et une entrée pomme de terre. Mais que faire alors des pommes vapeur et des pommes frites? On ne peut

\footnotetext{
6 Sur les rapports entre dictionnaire et idéologie (et notamment les tabous sexuels), cf. Domenico D'Oria (1998).

$7 \quad$ Cité dans : de Sepausy, V. «Le Petit Robert 2009 : toujours un grand pas pour la langue !». Actuallite.com [en ligne] <http://www.actualitte.com/actualite/2872-Le-Petit-Robert-2009dictionnaire.htm> consult.[10/06/2009]).
} 
les introduire ni sous l'entrée pomme - impossibilité sémantique -, ni sous l'entrée pomme de terre - impossibilité formelle ; une solution, dès lors, est de créer deux entrées homonymes pomme (dont l'une est une ellipse de pomme de terre). Mais si l'on admet comme entrée la lexie complexe pomme de terre, pourquoi ne pas admettre aussi pomme de pin ? Pomme d'Adam? Pomme d'arrosoir?

Une deuxième difficulté de microstructure consiste à choisir entre homonymie et polysémie: va-t-on par exemple considérer la lexie grève comme un mot polysémique (/terrain plat formé de sable/ vs. /cessation volontaire du travail/) ou comme deux mots homonymes ? L'étymologie pousse à opter pour la polysémie (la première grève ouvrière ayant eu lieu Place de Grève à Paris, dans une boucle de la Seine), mais une préférence synchronique favorise plutôt une présentation en deux termes homonymes. Les choix opérés par les dictionnaires sont en ce domaine parfois différents: une seule entrée bouton (de fleur, sur la joue, de chemise) dans le Robert mais trois dans le Larousse en ligne ; une entrée col dans le Larousse mais trois dans le Lexis. Certes, pour choisir entre homonymie et polysémie, le lexicographe dispose bien de connaissances étymologiques, mais, dans la mesure où la plupart des dictionnaires de langue se veulent synchroniques, il fait surtout appel à son «sens de la langue »; d'où une marge d'appréciation subjective, empirique, incertaine.

Troisième problème, celui de la structure globale du dictionnaire, sa macrostructure. L'organisation la plus fréquente est alphabétique; fondamentalement non sémantique, non étymologique et purement formelle, cette organisation n'est cependant pas la seule possible :

- l'ordre alphabétique s'applique ici aux seuls graphèmes, mais on pourrait envisager un dictionnaire qui classe alphabétiquement les phonèmes et permette ainsi de trouver facilement les mots dont on ignore l'orthographe ;

- le Grand Robert, dans son projet initial, devait être un dictionnaire analogique permettant de découvrir «le mot dont on a besoin ou qu'on a oublié » ${ }^{8} ;$ d'où une organisation onomasiologique complexe, qui n'a jamais été mise en œuvre finalement dans le Grand Robert ;

- dans sa première édition, le Dictionnaire de l'Académie française, dans une perspective étymologique, classait les mots par racine (cette organisation peu commode et élitiste a été par la suite délaissée au profit du strict classement alphabétique) :

- $\quad$ existent encore des solutions mixtes : le Dictionnaire du français contemporain puis le Lexis de Jean Dubois multiplient les homonymes et introduisent sous les entrées leurs dérivés morphologiques: on trouve par exemple les mots déchargement, surcharger, chargement sous l'entrée charger; l'ordre alphabétique s'en trouve altéré mais la cohérence sémantique renforcée.

Cité par Josette Rey-Debove (2003 :100).

Les Cahiers de l'APLIUT - Vol. XXIX Nº 1 - FÉVRIER 2010 - ISSN 0248-9430 
On le constate à travers ces trois problèmes - et il y en aurait bien d'autres ! -, les choix qui s'offrent au dictionnariste en matière de structure sont multiples et complexes; d'un dictionnaire à l'autre, l'organisation des entrées, leur présentation, leur regroupement sont susceptibles de varier et exigent en tout cas autant d'ingéniosité que de rigueur : en ce domaine aussi, le dictionnaire apparaît bien, ici encore, comme « un concurrent naïf, pesant, bricolé, ingénieux, empirique, impur, du texte scientifique, produit faussement neutre de la réflexion scientifique » (A. Rey $1977: 82$ ).

\section{Définir}

Définir un mot est un exercice extrêmement complexe qui implique des problèmes philosophiques, logiques, linguistiques que nous ne pouvons ici développer. Dans un dictionnaire, la définition est le plus souvent constituée d'une périphrase synonymique, ce qui entraîne un phénomène de circularité ${ }^{9}$ puisqu'on tente de définir un concept par une série de signes désignant eux-mêmes des concepts : à cette difficulté logique s'ajoutent des obstacles pratiques : comment par exemple induire de discours particuliers des significations générales en langue ?

On ne prendra ici que quelques exemples montrant combien l'exercice est périlleux... En mai 2006, lors de la sortie du Robert 2007, naît une polémique enflammée sur le mot colonisation défini (depuis la première édition du Robert en 1967) comme une «Mise en valeur, exploitation des pays devenus colonies»; le $M R A P^{I 0}$ met en avant que la définition tait les aspects négatifs de la colonisation et demande le retrait pur et simple de l'édition 2007. À quoi Alain Rey répond par le fait que valeur a un sens monétaire ${ }^{11}$; et d'ajouter: "Au-delà de ça, si on n'a pas le droit de parler des côtés positifs d'une chose qui est globalement négative, c'est une forme de révisionnisme!». Cet exemple est révélateur à plusieurs titres : d'abord, il nous montre toute l'importance que l'on attribue aux mots et à leurs définitions, particulièrement quand elles figurent dans des dictionnaires de référence (ce qui se joue ici déborde du cadre linguistique pour envahir le terrain social et idéologique); d'autre part, il nous montre la pression exercée par la société sur le lexicographe : dans la mesure où il est considéré - à tort - comme prescriptif, le dictionnaire a un devoir de réserve et de neutralité auquel les lobbies et groupes de pression de toutes sortes seront particulièrement sensibles.

\footnotetext{
$9 \quad$ Sur la circularité, cf. par exemple Collinot (1997: 165) ou Hagège (2009 : 90).

10 Mouvement contre le Racisme et pour l'Amitié entre les Peuples.

11 «C'est un terme qui relève de la sphère financière, qui n'a en soi pas de connotation positive ou négative. La mise en valeur d'une station de sports d'hiver ne veut pas dire qu'on va s'occuper de la nature, mais qu'on l'aménage pour se faire du fric ! Et qu'était la colonisation de nouvelles terres sinon l'exploitation, la mise en valeur de ses richesses, au bénéfice des colons ? » cité dans Libération $\quad<$ http://www.liberation.fr/societe/010113927-colonisation-alain-rey-contre-1inculture-economique $>$. (consult. [10/06/2009]).
} 
De ce fait, le lexicographe tend à tenir le discours attendu de lui par ses contemporains, en fonction des préjugés de son temps : la définition apparaît bien comme le produit d'une époque. Examinons par exemple l'évolution du mot femme à travers quelques dictionnaires : derrière les définitions, les exemples et les citations, c'est bien le statut de la femme, et l'histoire de son émancipation que l'on voit transparaître.

\section{Dictionnaire de l'Académie française, $1^{\mathrm{e}}$ Edition (1694)}

femme. s. f. La femelle de l'homme. Dieu tira la femme de la coste d'Adam. les femmes sont naturellement timides. il y a plus de femmes que d'hommes dans une telle ville. cet homme est adonné au vin \& aux femmes. la frequentation des femmes debauchées est fort dangereuse. femme mariée.

Femme, se dit aussi, pour signifier Celle qui est, ou qui a esté mariée. Et en ce sens il est opposé à Fille. Les femmes \& les filles. femme en puissance de mari. mari \& femme. femme sage. femme de bien. honneste fermme. fermme grosse. femme veuve. c'est sa legitime femme. femme separée de son mari. femme authorisée en Justice.

On dit prov. Appetit de femme grosse, pour dire, Un appetit dereglé. Et, Ce que femme veut Dieu le veut, pour dire, que Les femmes veulent fortement ce qu'elles veulent. Et prov. aussi, Quand il pleut \& qu'il fait soleil en mesme temps. On dit que Le diable bat sa femme.

On appelle, Femme de chambre, ou absolument Femme, Une femme ou fille qui sert une Dame à la chambre. Et, Femme de charge, Celle qui a soin du linge, de la vaisselle d'argent \&c.

Sage-femme. s. f. Matrone, Celle qui aide les femmes à accoucher. Chercher une sage-femme. envoyer querir une sage-femme

\section{Nouveau Petit Larousse illustré (1956)}

femme [fam.] n. f. (lat. femina). Compagne de l'homme; épouse. Celle qui est ou a été mariée. Femme de chambre, femme attachée au service intérieur d'une personne de son sexe. Femme de charge, celle qui a soin du linge, de l'argenterie, etc. d'une maison. Femme de ménage, femme chargée du soin d'un ménage dans une famille, et qui est payée à l'heure ou à la journée. Bonne femme, femme âgée, femme sans prétentions.

\section{Petit Larousse en couleurs (1988)}

femme. [fam.] n. f. (lat. femina). 1. Être humain du sexe féminin (par opp. à homme). La loi salique excluait les femmes de la possession de la terre. 2. Adulte du sexe féminin (par opp. à fille, à jeune fille). C'est une femme maintenant. 3. Épouse. Il nous a présenté sa femme. 4. (Qualifié). Adulte du sexe féminin considéré par rapport à ses qualités, ses défauts, ses activités, ses origines, etc. Une brave femme. Une femme de parole. Une femme de lettres. [ ... ]

\section{Petit Larousse (en ligne )}

femme nom féminin (latin femina)

-Être humain appartenant au sexe apte à reproduire des ovules et à porter des enfants ; être humain femelle.

-Adulte de sexe féminin, par opposition à fille, jeune fille, et, en particulier, personne de sexe féminin nubile ou ayant perdu sa virginité : La voilà une femme maintenant.

-Épouse : Il nous a présenté sa femme. 
-Adulte de sexe féminin, considéré par rapport à ses qualités, à ses défauts, à son activité, à son origine, etc. : Femme du monde. Femme politicienne.

-Peut être suivi ou précédé d'un nom de profession ou de fonction de genre masculin : Une femme ingénieur. Un professeur femme.

-Suivi d'un nom apposé, désigne une personne de sexe féminin qui a toutes les caractéristiques impliquées par le sens du nom apposé : Une femme enfant.

Les relations langue/société peuvent aussi s'exercer au niveau même de la conception de la description lexicographique : prenons ainsi l'exemple du mot chat que nous empruntons encore à Alain Rey (1977: 108).

\author{
Richelet (1690) \\ CHAT : Animal très connu, qui est ordinairement gris ou noir ou noir, gris et blanc, \\ ou noir et blanc, qui a les yeux étincelants, qui est fin, qui vit de souris et de toute \\ sorte de chair; qui hait les rats, les souris, les chiens, les aigles, les serpents et l'herbe \\ qu'on appelle la rüe. »

\section{L'Encyclopédie (1751)} \\ CHAT, s. m. felis, catus, (Hist. nat.) animal quadrupède domestique, dont on a donné \\ le nom à un genre de quadrupèdes, felinum genus, qui comprend avec le chat des \\ animaux très - sauvages \& très féroces. Celui - ci a sans doute été préféré dans la \\ dénomination, parce qu'y étant le mieux connu, il étoit le plus propre à servir d'objet \\ de comparaison pour donner quelques idées du lion, du tigre, du léopard, de l'ours, à \\ ceux qui n'en auroient jamais vû.

\section{Littré (1870)} \\ CHAT : Animal domestique, de l'ordre des carnassiers digitigrades. \\ Dictionnaire de L'Académie française, $\mathbf{8}^{\mathrm{e}}$ Edition (1932-1935) \\ CHAT : Animal domestique de l'ordre des Carnassiers et de la famille des Félins.

\section{Robert (1993)} \\ CHAT : \\ 1-Petit mammifère familier à poils doux , aux yeux oblongs et brillants, à oreilles \\ triangulaires et griffes rétractiles, qui est un animal de compagnie. \\ 2- Personne qui poursuit les autres \\ 3- Zool Mammifère carnivore dont le chat(1) est le type \\ Larousse (2008) \\ CHAT: Mammifère carnivore (félidé), sauvage ou domestique, au \\ museau court et arrondi.
}

Les deux premiers dictionnaires définissent l'animal en en proposant une évocation familière et descriptive, à l'aide de quelques éléments choisis arbitrairement. À partir du $19^{\mathrm{e}}$ siècle au contraire, la définition se fait scientifique, le chat étant défini comme hyponyme d'un hyperonyme donné (quadrupède, carnassier, mammifère) : mais si la précision taxinomique est juste, elle permet difficilement au lecteur non scientifique de concevoir à quoi peut bien ressembler un chat! Aussi les deux 
derniers dictionnaires associent-ils aux précisions zoologiques des détails descriptifs permettant à chacun d'y trouver son compte.

Mais comment extraire ces indices ? Quelles sont les caractéristiques les plus significatives permettant de distinguer le chat de la belette ou de la souris ? On le voit, là encore l'approximation, l'arbitraire et la démarche empirique sont finalement la règle.

On voit bien ainsi la difficulté de cette entreprise définitionnelle : car, au-delà des problèmes lexicographiques et logiques, se profile en effet, derrière le mot, le référent et donc la société de l'époque avec ses tabous et ses préjugés. Chercher à y échapper et à respecter ainsi la devise de Paul Robert «Tout le défini, rien que le défini $»^{12}$ est certes louable mais demeure en pratique bien souvent un vœu pieux.

\section{Illustrer}

Les illustrations, qui peuvent apparaître sous la forme de dessins, photos mais aussi de citations ou d'exemples, servent ou bien à expliciter la définition, ou bien, à l'époque classique, à proposer des modèles à suivre, ou bien encore à attester de la réalité de ce qu'on affirme (modèle positiviste) : réinsérer la définition dans le discours permet en effet à la fois de justifier la nomenclature et de redonner une réalité au mot.

L'une des méthodes en ce domaine consiste, comme le pratique par exemple le Dictionnaire de L'Académie française, à forger les exemples pour les besoins de la cause, sans se référer à des citations extraites de tel ou tel corpus. On pourra contester l'intérêt d'une telle pratique qui prive le mot de son insertion discursive : au moins s'épargne-t-on des polémiques, comme celle qui, à nouveau, prit pour cible Le Robert édition 2008. C'est une citation de Jean-Claude Izzo, destinée à illustrer le mot rebeu qui cette fois déclencha l'ire de l'Unsa-Police: "T'es un pauvre petit rebeu qu'un connard de flic fait chier. C'est ça! ${ }^{13}$ Aussitôt, le syndicat d'appeler officiellement les 150000 fonctionnaires de police au boycott du Petit Robert édition 2008 ; une action en justice est déposée afin d'obtenir le retrait de l'illustration contestée. "Il est inadmissible qu'une institution comme la police, qui porte aide et assistance à la société, soit ainsi bafouée » déclare ainsi Joaquin Masanet, secrétaire général de l'Unsa-Police. A quoi les éditions Le Robert rétorquent: «La mission du dictionnaire [...] n'est pas de 'jeter le discrédit et le déshonneur' sur quiconque, mais de décrire la langue dans toute sa richesse et ses multiples usages, du plus soutenu au plus familier ». Alors, faut-il choisir plutôt de forger ses propres exemples et citations ? On tend alors vers le prototype, voire vers le stéréotype qui ignore la variété - parfois dérangeante - des situations énonciatives.

12 Cité par J. Rey-Debove (2003:105).

13 Le Robert 2008, page 2135 ; citation extraite de Total Kheops de Jean-Claude Izzo (1996).

Les Cahiers de l'APLIUT - Vol. XXIX Nº 1 - FÉVRIER 2010 - ISSN 0248-9430 
Le problème, on le voit là encore, c'est que parler des mots, c'est parler du monde : le dictionnaire n'est pas qu'un outil de description du code linguistique, il est toujours perçu comme cautionnant ou proposant une certaine vision du monde. L'illustration ne saurait donc apparaître comme totalement «innocente »; on en trouverait une nouvelle preuve en examinant l'iconographie du dictionnaire Mon Larousse en images (1956) : on y verrait transparaître les stéréotypes de l'époque, une société qui oppose à des jeunes filles fragiles, émotives et coquettes, à des femmes essentiellement préoccupées des soins du ménage, des hommes qui travaillent et savent se faire obéir.

\section{Conclusion}

Le dictionnaire de langue est souvent vu comme un outil indiscutable, anonyme, qui nous dit l'immuable vérité des mots. Dans la réalité, le dictionnaire est un texte, un discours bricolé par des artisans de la langue ; à y regarder de plus près, en effet, il n'y a rien de donné, d'évident dans l'élaboration du dictionnaire : le travail du lexicographe consiste à faire des choix : dans l'établissement de la nomenclature, la structuration du dictionnaire (polysémie, homonymie), aussi bien que dans l'élaboration de la définition ou le choix des illustrations (textuelles ou graphiques), le lexicographe doit effectuer en permanence des ajustements, des approximations ; il est certes guidé par des règles scientifiques qu'il établit luimême, mais il doit aussi parfois s'en remettre à son sens de la langue pour faire des choix nécessairement subjectifs et discutables.

Un bricolage, disions-nous : bricolage à haut risque, car le dictionnaire est pris pour ce qu'il n'est pas, une référence prescriptive : on croit qu'il nous dit ce qu'il faut entendre, alors qu'il se contente de répertorier ce qu'on dit effectivement ; d'où les polémiques et procès que l'on voit se développer depuis quelque temps.

Un travail qui demeure imparfait, incertain, empreint par bien des côtés de subjectivité : non que ceux qui s'y adonnent manquent de compétences, mais parce qu'il est impossible de mener une entreprise rigoureusement scientifique sur la langue qui demeure un matériau humain, incomplet, mouvant.

Un travail qui demeure artisanal, et qui rejoint par là le bricolage cher à LéviStauss (1962: 32) : «Mais il y a plus : la poésie du bricolage lui vient aussi, et surtout, de ce qu'il ne se borne pas à accomplir ou exécuter ; il raconte [...] le caractère et la vie de son auteur. Sans jamais remplir son projet, le bricoleur y met toujours quelque chose de soi. »

\section{Bibliographie}

Collinot, A. \& F. Mazière. 1997. Un prêt à parler : le dictionnaire. Paris : PUF.

Cormier, M.C., A. Francoeur, J. Rey-Debove et alii. 2003. Les dictionnaires Le Robert : genèse et évolution. Montréal : PUM. 
d'Oria, D. 1998. Dictionnaire et idéologie. Paris : Schena-Nizet.

Delesalle, S. \& L. Valensi. 1972. « Le mot 'nègre' dans les dictionnaires français de l'Ancien Régime : histoire et lexicographie ». Langue française vol n 15 : 79-104.

Girardin, C. 1979. «Contenu, usage social et interdits dans le dictionnaire». Langue Française vol n`43 : 84-99.

Hagège, C. 2009. Dictionnaire amoureux des Langues. Paris : Plon.

Mortureux, M.-F. 1997. La lexicologie entre langue et discours. Paris : Sedes.

Pottier, B. 1974. Linguistique générale : théorie et description. Paris : Klincksieck.

Pruvost, J. 2002. Les dictionnaires de langue française. Paris : PUF (Que sais-je).

Rey, A. 1977. Le lexique: images et modèles. Du dictionnaire à la lexicologie. Paris : Colin.

Rey, A. 2010. Dictionnaire amoureux des dictionnaires. Paris : Plon.

\section{Bibliographie complémentaire}

Barthes, R. 1985. L'aventure sémiologique. Paris : Seuil.

Lévi-Strauss, C. 1962. La pensée sauvage. Paris : Plon.

Perec, G. 1989. L'infra-ordinaire. Paris : Seuil (La Librairie du XX $X^{\mathrm{e}}$ siècle). 